Review paper

\title{
Recommendations for the use of active personal dosemeters (APDs) in interventional workplaces in hospitals
}

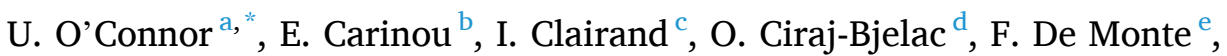 \\ J. Domienik-Andrzejewska ${ }^{f}$, P. Ferrari ${ }^{g}$, M. Ginjaume ${ }^{h}, H$. Hršak $^{i}$, O. Hupe $^{j}$, Ž. Knežević $^{k}$, \\ M. Sans Merce ${ }^{1, m}$, S. Sarmento ${ }^{n}$, T. Siiskonen $^{\circ}$, F. Vanhavere $^{\mathrm{p}}$ \\ a St. James's Hospital, Dublin, Ireland \\ ${ }^{\mathrm{b}}$ Greek Atomic Energy Commission, EEAE, Agia Paraskevi, Attiki, Greece \\ ${ }^{\mathrm{c}}$ IRSN, Institute for Radiological Protection and Nuclear Safety, Fontenay-aux-Roses, France \\ ${ }^{\mathrm{d}}$ Vinca Institute of Nuclear Sciences, University of Belgrade, Belgrade, Serbia \\ ${ }^{\mathrm{e}}$ Veneto Institute of Oncology IOV_IRCCS, Padua, Italy \\ ${ }^{\mathrm{f}}$ NIOM, Nofer Institute of Occupational Medicine, Lodz, Poland \\ ${ }^{g}$ ENEA. IRP - Radiation Protection Institute, Bologna, Italy \\ ${ }^{\text {h }}$ UPC, Universitat Politècnica de Catalunya, Barcelona, Spain \\ ${ }^{\mathrm{i}}$ University Hospital Centre Zagreb, Croatia \\ ${ }^{\mathrm{j}}$ Physikalisch-Technische Bundesanstalt (PTB), 38116 Braunschweig, Germany \\ ${ }^{\mathrm{k}}$ Ruđer Bošković Institute RBI, Zagreb, Croatia \\ ${ }^{1}$ CHUV, University Hospital of Lausanne, Lausanne, Switzerland \\ ${ }^{\mathrm{m}}$ HUG, University Hospital of Geneva, Geneva, Switzerland \\ ${ }^{\mathrm{n}}$ IPO Porto Research Center (CI-IPOP), Portuguese Oncology Institute of Porto (IPO Porto), Porto, Portugal \\ o STUK, Helsinki, Finland \\ p SCK-CEN, Belgian Nuclear Research Centre, Mol, Belgium
}

\section{A R T I C L E I N F O}

\section{Keywords:}

Radiation

Protection

Dosemeter

Interventional

\begin{abstract}
A B S T R A C T
Occupational radiation doses from interventional procedures have the potential to be relatively high. The requirement to optimise these doses encourages the use of electronic or active personal dosimeters (APDs) which are now increasingly used in hospitals. They are typically used in tandem with a routine passive dosimetry monitoring programme, with APDs used for real-time readings, for training purposes and when new imaging technology is introduced. However, there are limitations when using APDs. A survey in hospitals to identify issues related to the use of APDs was recently completed, along with an extensive series of APD tests by the EURADOS Working Group 12 on Dosimetry for Medical Imaging. The aim of this review paper is to summarise the state of the art regarding the use of APDs. We also used the results of our survey and our tests to develop a set of recommendations for the use of APDs in the clinical interventional radiology/cardiology settings, and draw attention to some of the current challenges.
\end{abstract}

\section{Introduction}

The European Radiation Dosimetry (EURADOS) group is a non-profit association for promoting research and development and European cooperation in the field of the dosimetry of ionizing radiation. The network of 80 European institutions includes experts, reference and research laboratories, and dosimetry services. Much of the work is performed in working groups (WGs) on dosimetry related topics. WG12 is focussed on dosimetry in medical imaging, covering both occupational and patient exposure. A key activity of WG12 in recent years has been investigating the use of active personal dosemeters (APDs) in hospitals. The aim of this paper is to summarise the significant body of research done on this topic, starting with the relevant standards and literature and concluding with recommendations for the use of APDs, with particular focus on their use during interventional procedures.

\footnotetext{
* Corresponding author at: Medical Physics \& Bioengineering Department, Hospital 1, St. James's Hospital, Dublin 8, Ireland.

E-mail address: uoconnor@stjames.ie (U. O’Connor).
} 


\subsection{Standards and guidance on occupational monitoring, including APDs}

The IAEA Basic Safety Standards state that assessment of the occupational exposure of workers on the basis of individual monitoring shall be made with authorized or approved dosimetry service providers that operate under a quality management system [1]. The EU Basic Safety Standards state that Member States shall ensure that dosimetry services determine internal and external doses to exposed workers subject to individual monitoring, and that arrangements must be in place for the approval of such dosimetry services [2]. Workplace monitoring programmes should be established in consultation with a qualified expert; the Basic Safety Standards do not specify the frequency of monitoring or type of dosimeter.

Guidance on the use of APDs is available in the IAEA General Safety Guide GSG-7 [3]. This guide states that APDs can be useful for optimisation and that they can be recommended for specific purposes, such as short term radiation control of workers' exposures, or during a particular task. An active dosemeter might also serve the purpose of maintaining alertness to possible accidental exposures. It can also be used for dose control in situations where the radiation field experienced by a worker could increase unexpectedly and significantly (say, by a factor of ten), or for operations of short duration in high radiation fields. In the same Safety Guide it is also stated that while an APD is usually used only for purposes of dose control, it can also be used with prior approval from the regulatory body, as a replacement for the dosemeter of record. In such cases, the same approval procedures by the regulatory body should apply. The active dosemeter should be of a suitable design for use as the dosemeter of record. It should have, for instance, an adequate energy range, sensitivity, linearity and precision; it should be reliable; and sufficient quality control measures and periodic calibration procedures should be in place.

Similar guidelines can be found in the European Commission RP160 recommendations [4]. APDs should be used when it is necessary to control individual exposure on a day to day basis or when the radiation field experienced by a worker could increase significantly and unexpectedly. Active and passive dosemeters are now frequently used together, the former for its direct reading capability and the latter for regulatory monitoring of occupational exposure. It is noted here that the use of APDs is evolving from being work control devices, to fulfilling all the legal aspects of individual monitoring.

Finally, ICRP Report 139 states that active, electronic personal dosemeters have proven useful for optimisation monitoring in interventional procedures and for special studies of dose per procedure [5]. Use of real-time active dosemeters not only helps in optimising protection of specific high-dose procedures, but also contributes to the education of professionals on the level of doses being received. APDs are able to provide immediate information about dose rate and this information is needed if actions are desired during a procedure, as it can lead directly to action by staff. In addition, some models of APDs can provide information on the time of each exposure, which facilitates correlation of occupational and patient exposures, and auditing of the wearing of the APD during interventions.

\subsection{Study background and aim of review}

A previous study which was based on a questionnaire to the users of APDs in industry and hospitals [6,7] showed that at that time, around 15 years ago, APDs were rarely used in hospitals. Also, at this time, an intercomparison of APDs was organized as a joint venture project of EURADOS and the International Atomic Energy Agency (IAEA) to assess the technical capabilities of different types of APDs. This key report showed that the general performance of the APDs was comparable with standard passive dosemeters [8].

The growing use of APDs in hospitals across the EU was studied extensively by the ORAMED (Optimization of RAdiation protection of MEDical staff) project which concluded in 2011. A range of commercially available APDs were tested in laboratory and clinical conditions and a series of useful guidelines for APDs in interventional cardiology (IC) and interventional radiology (IR) workplaces were prepared [9-11]. A key finding was that there are sufficient APDs that have satisfactory energy and angular responses. However, in pulsed radiation fields, the response of most APDs decreases when the instantaneous personal dose equivalent rate increases [12]. The ORAMED report includes a summary of recommendations on selecting and using APDs in IR/IC. When selecting an APD, it must fulfil the requirements of the IEC 61526 (2010) standard, with particular focus on the energy response, angular response, maximum dose equivalent rate and response in pulsed radiation fields [13]. When using an APD, the ORAMED report made several practical recommendations including that the device must be calibrated periodically in a laboratory traceable to a primary standard and that it should be worn over the lead apron. Finally, it was recommended that the acoustic alarm should be switched off; only the visual alarm should be used for warning operators when they are too close to the direct beam [12].

In more recent years, new designs of APDs have become available, and there have been publications on the standardization of tests for pulsed radiation fields $[14,15]$. Advances in battery power management and wireless transmission (e.g. from an APD to a base station) have overcome some of the disadvantages of using electronic dosemeters. Some manufacturers of IR/IC systems have developed TV monitors with wireless data transmission, so that staff doses can be viewed in real-time as part of the X-ray image screen [5]. A subsequent increase in the use of APDs in hospitals was apparent and the use of ionizing radiation in medicine was continuing to rapidly develop in terms of technical complexity of IR/IC procedures.

With this in mind, a new task was identified by EURADOS WG12 and a series of actions were undertaken. These actions included an extensive survey in hospitals to identify issues related to APDs in medical applications [16], an assessment of the influence of lead aprons on the calibration and use of APDs and passive dosemeters [17] and tests on APDs in standard continuous and pulsed fields according to the new ISO technical specification [18]. Finally, a task in two parts was completed in hospitals to assess the performance of APDs in the clinical environment. The response of APDs was tested compared to reference measurements in realistic hospital fields using interventional equipment (paper in preparation); and selected APDs were worn together with a passive dosemeter during interventional procedures by medical staff in several European hospitals [19]. Further details on the make/model of APDs that were tested can be found in the above referenced series of papers published by EURADOS WG12. The ultimate aim of this coordinated set of actions was to formulate updated recommendations on the use of APDs in hospitals, where guidance on their use (particularly in pulsed fields) is needed. This review paper gives an overview of the findings of these actions, and lists the recommendations resulting from the experience.

\section{Trends in the usage of APDs}

Our review of the literature has highlighted that in the early 2000s, APDs were rarely used in hospitals $[6,7]$ but the landscape in the medical sector is clearly changing. The use of interventional radiology is increasing, and this raises new issues in terms of occupational protection in medicine [5]. APDs are increasingly used in various fields of application of ionising radiation in medicine (radiotherapy, nuclear medicine, IR/IC) and there is now regular use of APDs in European hospitals $[16,19,20]$. A 2004 survey [6] on APD use was addressed to Radiation Protection Officers (RPOs) in different institutes including nuclear power plants (NPP), hospitals, military, industrial and research settings. Seven out of $39(18 \%)$ responses were from users in the medical field and these seven respondents had only a limited number of APDs in use. This is in comparison to the NPP sector where at some sites over 1,000 APD units were in use. At that time, the use of APDs in the medical sector 
was mainly for pilot studies; routine use was limited. The reasons given were related to cost, size of APDs, battery life and electromagnetic compatibility.

A recent survey in 2018 [16] conducted by EURADOS WG12 was focused on the medical sector only as it was hypothesised that usage of APDs in this field has increased over the years. The survey was distributed through the EURADOS network to medical physicists and hospital RPOs. 74 responses were analysed from 19 different European countries. Of these, 50 (68\%) reported regular or occasional use of APDs. $40 \%$ of the responses are related to the use of a relatively small number of dosimeters $(<10)$. The largest number of dosimeters $(>50)$ was linked to routine use in interventional radiology and nuclear medicine departments in large teaching hospitals (in France and Germany). A small number of APDs $(<5)$ was mainly reported in smaller departments in which these dosimeters are used for research purposes. Although the target audience or end-users of the 2004 and 2018 surveys were different, with the more recent survey addressed to the medical sector only, it can be seen that the overall usage in hospitals has increased over the period.

This increased use of APDs is a good fit for a generation of personnel who have rapid access to information at their fingertips with smartphones. Workers are typically more interested and aware of their radiation dose records if it is instantly available. Rapid feedback of doses to staff can encourage changes in behaviour that are beneficial in terms of radiation protection. APDs are mainly used as a tool when new techniques are introduced, or in areas where there is potential for high levels of scattered radiation. They are also used for optimising protection and training staff $[5,21]$. The relatively high unit cost, combined with a lack of approved APDs (as a dosemeter for monitoring compliance with regulatory dose limits) in some countries, means however that APDs are still rarely used as the sole dosemeter.

For interventional procedures, passive dosimetry systems remain the most widely used option in hospitals to verify compliance with regulatory dose limits [5]. Passive dosemeters are small, lightweight, do not require power and can be worn in small packages that do not interfere with the staff's actions and comfort. Depending on the monitoring programme in place, staff may be issued with one or two passive wholebody dosemeters (for over/under the lead apron), an additional dedicated eye lens dosemeter, and where the dose to the hands requires monitoring, small ring-shaped dosimeters can be used. Due to the number of dosemeters, the complexity of positioning them, and other challenges described in the next section, it is unlikely that passive dosimetry will be fully replaced with APDs. Individual APD units are expensive and the costs would generally be prohibitive for all the staff monitoring requirements in most diagnostic imaging departments. In addition, for extremity monitoring, APDs have not been widely developed for routine use on the fingers or near the eyes. An attempt was made to place small electronic sensors on the fingers, but the electrical cables leading back to the power source and electronics were not convenient or practical for routine use [5].

Nonetheless, it is recognised that for dose optimisation, for teaching and training, and for experienced staff who may be adapting to work with new technologies, the complementary use of APDs can prove to be very useful as a dose-awareness tool $[20,22]$. With passive dosimetry the absence of an instant reading and alarm functions is a disadvantage, especially for the training of workers involved in interventions [5]. The minimum detection limit is also generally lower (more sensitive) for APDs than for passive dosimeters [7]. For many of the reasons described here, the use of real-time dosimetry with APDs continues to increase at the current time.

\section{Technical aspects of APDs and ongoing challenges}

As introduced above, the use of APDs is now widespread in hospitals and is generally found to be beneficial. A note of caution remains, however, due to limitations that should be considered and communicated to hospital users to raise awareness when selecting or using APDs. Many of the challenges with the use of APDs have been discussed in the aforementioned WG12 series of publications. The WG12 work concluded that in the majority of cases, calibration of APDs is not adequately addressed. Guidance from regulatory authorities, the metrology community and professional organizations on testing and calibration of APDs used in hospitals is needed [16].

The IEC 61526 standard [13] specifies general characteristics, test procedures, radiation characteristics as well as electrical, mechanical, safety and environmental characteristics for APD type testing. The type testing of APDs should be done with reference radiation qualities according to ISO 4037-1 [23]. Performing the type test with the narrowseries radiation quality will ensure a satisfactory energy response in realistic radiation fields (with a broader energy distribution). In cases where a dosemeter is used only in a specific workplace with a wellknown radiation field, it is possible to calibrate the dosemeter at a reference radiation quality similar to that of the workplace. However, in general, the use of a calibration factor for the APD from a workplacelinked radiation quality is not needed, considering the uncertainties usually required for radiation protection dosimetry. When an APD is used as an official dosemeter, testing for protection against data manipulation and software security (both the read-out software and the dosimeter firmware) must be done. Requirements for this can be found in WELMEC Guide 7.2 [24].

There are concerns with the response of APDs in pulsed fields. The IEC 61526 standard [13] does not cover the special requirements for pulsed radiation. Thus, when used in IR/IC fields, APDs have to comply with additional tests as described in the recent IEC/TS 63050 [14] using reference radiation according to ISO/TS 18090-1 [15]. This is the first standard that specifically addresses the special characteristics required for dosemeters to be used in pulsed fields and a useful summary on the latest updates to the IEC standards for active dosemeters is available [25]. The available data from manufacturers on pulsed field response is often limited. In particular, most data on dose rate dependence is valid only for continuous radiation fields. The performance of the dosemeter determined in continuous radiation fields cannot be transferred to pulsed fields. Therefore, it is vital to increase awareness amongst users, and manufacturers, that if the dosemeter is to be used in pulsed fields, the dosemeter may indicate incorrect values, in most cases lower, than that measured with a passive dosemeter [18].

There is also a need to give attention to the appropriate energy range of the APDs, especially for low energies $(<60 \mathrm{keV})$ which is relevant for scattered radiation in the interventional setting. It has also been determined that comparing active and passive dosemeters is not straightforward in clinical settings for numerous reasons, and the relative position of the dosemeter on the operator can be a significant influence [19].

For all APDs, the dose can be significantly underestimated if it is placed in the direct beam. This would not be expected routinely as workers are reminded to increase their distance as much as possible from the patient. However, it is a possible rare event that an APD on the chest of an operator could be very close to the primary beam if the doctor is leaning over the patient. The dosemeter should be able to function in these conditions and there is a need for visual alarms if the APD is overloaded or saturated.

Bearing in mind the response required in these situations, it is useful to have a reference range of typical radiation fields encountered in interventional workplaces including the pulse duration, pulse frequency 
Table 1

Typical radiation field characteristics in interventional radiology and cardiology workplaces (reproduced from [12]).

\begin{tabular}{ll}
\hline Parameter & Range \\
\hline Peak high voltage & $60-120 \mathrm{kV}$ \\
X-ray tube current & $5-1000 \mathrm{~mA}$ \\
Inherent Al equivalent filtration & $4.5 \mathrm{~mm}$ \\
Additional Cu filtration & $0.2-0.9 \mathrm{~mm}$ \\
Pulse duration & $1-20 \mathrm{~ms}$ \\
Pulse frequency & $1-30 \mathrm{~s}^{-1}$ \\
Personal dose equivalent rate integrated over the pulse & $2-300 \mathrm{~Sv} \cdot \mathrm{h}^{-1}$ \\
$\quad$ in the direct beam (on patient table) & \\
Personal dose equivalent rate in the scattered beam & $5.10^{-3}-10 \mathrm{~Sv}^{-1} \mathrm{~h}^{-1}$ \\
$\quad$ (operator - above the lead apron)* & \\
Average energy range of scattered spectra & $20-100 \mathrm{keV}$ \\
\hline
\end{tabular}

* distance patient-operator $=30 \mathrm{~cm}$.

and personal dose equivalent rates in both the direct and scattered beam, as shown below in Table 1 [12].

\section{Recommendations}

The following recommendations are intended as a practical aid to hospitals when selecting and using APDs for use in the IR/IC setting. It can be seen from the recommendations that not all challenges have been adequately met with the current state-of-the-art APDs, and challenges remain which are highlighted in the list below:

1. Standards. A core recommendation is that the APD should meet all the requirements of the relevant IEC standards including those for pulsed fields [14].

2. Legal approval. In principle, APDs can be approved as a dosemeter for regulatory compliance with dose limits or for dose record keeping, once the criteria required for approval have been set by the national framework, and fulfilled by the APD and dosimetry service.

3. Use with Passive Dosemeters. If passive dosemeters and APDs are used in parallel, it is crucial that the interpretation of results is carried out by a suitably qualified Radiation Protection Expert (RPE) familiar with properties of both types of dosemeters. Large uncertainties exist in personal dosimetry, and they can result in significant differences between two dosemeters worn by the same person. Characteristics of the types of dosemeters play a role here, but also the type of procedure and the position of the dosemeters on the worker, and the influence of lead aprons on dosemeters [17].

4. Instruction for Wearing and Use. Clear guidance and instruction should be provided to users in hospital on the use of APDs. It is recommended that they should be worn above the lead apron at chest height.

5. Calibration. Guidance from regulatory authorities on the periodic calibration of APDs, including the calibration radiation quality, should be developed.

6. Range of Operation and Product Evaluation. In general, as also applies to passive dosemeters, APDs should be acceptable in terms of their energy and angular response, sensitivity, linearity and accuracy. There is a need for expertise in the evaluation of APDs for use in hospitals, including input as appropriate from the RPO, the RPE, medical physicists, approved dosimetry services and manufacturers. The range in which the dosemeters fulfil the standards criteria should be carefully assessed and compared with the ranges in which they will be used in the hospitals. The scope of the use of APDs should also be established i.e. are they used for training or for the dose of record. Caution should be taken with the specifications listed by the manufacturers, which may be valid only in limited situations e.g. continuous fields. Particular care should be taken with low photon energies and large angles of incidence, as many dosemeters have a significant deviation in these cases and the response in pulsed radiation fields.

7. Pulsed Fields. If the dosemeter is to be used in pulsed fields the users must be aware that it may indicate incorrect values such as: large underestimations if placed in the direct beam, and a greater influence (again usually an underestimation) when the instantaneous dose rate is higher. Some local tests in the hospital comparing measurements from passive and active dosemeters could provide the user with information in this regard.

8. Alarms. APDs should include an alarm to indicate that the dosemeter is out of its range. Thus, the user will be alerted that something is wrong with the dosemeter or there is a possibility of increased radiation dose. It should not be an audible alarm (this should be switched OFF) and only the visual alarm should be used.

9. Software Options. Bearing in mind the requirements for data integrity above, software to manage a set of APDs is a useful tool and should be considered when acquiring dosemeters. There is risk with APDs of resetting the dose to zero, and of the battery failing mid-procedure, along with the potential for human error and the time required to manually transcribe data. Modern APDs that work with docking stations and download timestamped dose data can allow for more effective management in the hospital, and aid research and audit.

10. Data Integrity. The APD must be of a suitable design for use as the dosemeter of record, including the protection from data manipulation, software security, and protection against electromagnetic disturbances.

\section{Conclusion}

The use of APDs in hospitals is growing and there is clear interest from end-users in harnessing the benefits of real-time dosemeters. The EURADOS WG12 group has expanded on work done previously in this area, to perform a significant and detailed assessment of APDs in laboratory conditions and the clinical environment. Comparing active and passive dosemeters is not straightforward in the clinical setting where a range of energies, angles and pulsed field characteristics occur. APDs show consistently lower values than passive dosemeters during standard IR/IC procedures, and this must be considered when monitoring staff dose using pulsed radiation. The relative position on the body of the operator can also be a significant factor. Based on the work done on APDs, a series of recommendations have been made. It is hoped that they will be a practical guide to hospital users, medical physicists and radiation protection experts, and will make the use of APDs more reliable and meaningful. Future work should continue on the complex assessment of APDs in pulsed radiation fields, and the influence of the position of the dosemeter on the operator.

\section{Declaration of Competing Interest}

The authors declare that they have no known competing financial interests or personal relationships that could have appeared to influence the work reported in this paper.

\section{Acknowledgements}

Many thanks to all of the EURADOS WG12 members, hospital staff and laboratory staff that contributed to this series of tasks.

\section{References}

[1] IAEA. Radiation Protection and Safety of Radiation Sources: International Basic Safety Standards. IAEA Safety Series No. GSR Part 3. IAEA, Vienna; 2014.

[2] European Council Directive 2013/59/Euratom on basic safety standards for protection against the dangers arising from exposure to ionising radiation and 
repealing Directives 89/618/Euratom, 90/641/Euratom, 96/29/Euratom, 97/43/ Euratom and 2003/122/Euratom. OJ of the EU. 2014;L13; 57: 1-73.

[3] IAEA. Safety Standards Series No. GSG-7. Occupational Radiation Protection. General Safety Guide. IAEA, Vienna; 2018.

[4] European Commission (EC). Technical Recommendations for Monitoring Individuals Occupationally Exposed to External Radiation. RP 160. DirectorateGeneral for Energy and Transport. EC, Luxembourg; 2009.

[5] International Commission on Radiological Protection (ICRP). Occupational radiological protection in interventional procedures. ICRP Publication 139. Ann. ICRP 47(2); 2018.

[6] Bolognese T, Ginjaume M, Luszik-Bhadra M, Vanhavere F, Wahl W, Weeks A. Active personal dosemeters for individual monitoring and other new developments. Radiat Prot Dosim 2004;112(1):141-68. https://doi.org/10.1093/ $\mathrm{rpd} / \mathrm{nch} 286$.

[7] Ginjaume M, Bolognese T, Luszik-Bhadra M, Vanhavere F, Wahl W, Weeks A. Overview of active personal dosemeters for individual monitoring in the European Union. Radiat Prot Dosim 2007;125(1-4):261-6. https://doi.org/10.1093/rpd/ ncl136.

[8] International Atomic Energy Agency (IAEA). Intercomparison of Personal Dose Equivalent Measurements by Active Personal Dosimeters. IAEA-TECDOC-1564. ISBN 978-92-0-106607-7. IAEA, Vienna; 2007.

[9] Clairand I, Bordy J-M, Daures J, Debroas J, Denoziere M, Donadille L, et al. Active personal dosemeters in interventional radiology: tests in laboratory conditions and in hospitals. Radiat Prot Dosim 2011;144(1-4):453-8. https://doi.org/10.1093/ $\mathrm{rpd} / \mathrm{ncq} 556$.

[10] Struelens L, Carinou E, Clairand I, Donadille L, Ginjaume M, Koukorava C, et al. Use of active personal dosemeters in interventional radiology/cardiology: tests in hospitals. Radiat Meas 2011;46(11):1258-61. https://doi.org/10.1016/j. radmeas.2011.08.002.

[11] Clairand I, Bordy JM, Carinou E, Daures J, Debroasa J, Denozière M, et al. Use of active personal dosemeters in interventional radiology/cardiology: Tests in laboratory conditions and recommendations-ORAMED PROJECT. Radiat Meas 2011;46(11):1252-7. https://doi.org/10.1016/j.radmeas.2011.07.008.

[12] Vanhavere F, Carinou E, Gualdrini G, Clairand I, Sans Merce M, Ginjaume M et al. ORAMED: optimisation of RAdiation protection of MEDical staff. EURADOS Report. 2012-02. Braunschweig; 2012.

[13] International Electrotechnical Commission (IEC). Radiation protection instrumentation - measurement of personal dose equivalents $\mathrm{Hp}(10)$ and $\mathrm{Hp}(0,07)$ for X, gamma, neutron and beta radiations - direct reading personal dose equivalent meters. IEC 61526 Ed.3. IEC; 2010.
[14] IEC. Radiation protection instrumentation - Dosemeters for pulsed fields of ionizing radiation. IEC/TS 63050 Ed.1. IEC; 2019.

[15] International Organization for Standardization (ISO). Radiological protection—characteristics of reference pulsed radiation-Part 1: photon radiation. In: Technical Specification ISO/TS 18090-1. ISO; 2015.

[16] Ciraj-Bjelac O, Carinou E, Vanhavere F. Use of active personal dosemeters in hospitals: EURADOS survey. J Radiol Prot 2018;38:702. https://doi.org/10.1088/ 1361-6498/aabce1.

[17] Ginjaume M, Carinou E, Brodecki M, Clairand I, Domienik-Andrzejewska J, Exner L, et al. Effect of the radiation protective apron on the calibration of active and passive personal dosemeters used in interventional radiology and cardiology. J Radiol Prot 2019;39:97-112. https://doi.org/10.1088/1361-6498/aaf2c0.

[18] Hupe O, Friedrich S, Brodecki M, Vanhavere F. Determining the dose rate dependence of different active personal dosemeters in standardised pulsed and continuous radiation fields. Radiat Prot Dosim 2019;187(3):345-52. https://doi. org $/ 10.1093 / \mathrm{rpd} / \mathrm{ncz} 173$.

[19] Vanhavere F, Carinou E, Clairand I, Ciraj-Bjelac O, De Monte F, DomienikAndrzejewska $\mathrm{J}$ et al. The use of active personal dosemeters in interventional workplaces in hospitals. Comparison between active and passive dosemeters worn simultaneously by medical staff. Radiat Prot Dosim 2020; 188 (1) 22-9. https:// doi.org/10.1093/rpd/ncz253.

[20] Sanchez R, Vano E, Fernandez JM, Gallego JJ. Staff radiation doses in a real-time display inside the angiography room. Cardiovasc Intervent Radiol 2010;33(6): 1210-4. https://doi.org/10.1007/s00270-010-9945-4.

[21] Ginjaume M. Performance and approval procedures for active personal dosemeters. Radiat Prot Dosim 2011;144(1-4):144-9. https://doi.org/10.1093/rpd/ncq457.

[22] Martin CJ, Temperton DH, Jupp T, Hughes A. IPEM topical report: personal dose monitoring requirements in healthcare. Phys Med Biol 2019;64(3):035008. https://doi.org/10.1088/1361-6560/aafa3f.

[23] ISO. Radiological protection $-\mathrm{X}$ and gamma reference radiation for calibrating dosemeters and doserate meters and for determining their response as a function of photon energy - Part 1: Radiation characteristics and production methods. ISO 4037-1 Ed.2. ISO, 2019.

[24] WELMEC 7.2. European cooperation in legal metrology. Software Guide. (Measuring Instruments Directive 2014/32/EU1). WELMEC; 2019.

[25] Voytchev M, Behrens R, Radev R. Latest updates for the IEC standards for active and passive dosemeters. Rad Phys Chem 2020;166:108509. https://doi.org/ 10.1016/j.radphyschem.2019.108509. 